\title{
High Tibial Osteotomy Combined with Anterior Cruciate Ligament Reconstruction
}

\author{
Mohammed Alnahas* \\ Orthopedic Department, Faculty of Medicine, AL-Azhar University, Egypt
}

Submission: July 11, 2019; Published: July 25, 2019

*Corresponding author: Mohammed Alnahas, Orthopedic Department, Faculty of Medicine, AL-Azhar University, Nasr City, postal code: 11884 -Cairo, Egypt

\begin{abstract}
The presence of varus deformity in anterior cruciate ligament (ACL)-deficient knees can be a source of persistent knee pain owing to medial compartment overload. This deformity may threaten the outcome of ACL reconstruction because of increased tension on the graft. In the period between May 2018 to April 2019, short term pilot study including 10 patients with ACL deficiency and genu varus were treated by anatomical single bundle ACL reconstruction and open medial wedge high tibial osteotomy. The selected patients had manifestations of ACL deficiency in association with medial joint line pain with or without lateral thrust. Prospective evaluation using the international knee documentation committee score was performed. The mean follow-up period was 6 months. This technique achieved knee stability and realignment to normal values. The score significantly improved from 37 preoperative to 78 postoperative $(p<0.05)$. The combined procedure of ACL reconstruction and high tibial osteotomy restored knee stability and reduced pain over the medial compartment. Although the combined procedure has a longer period of rehabilitation than an isolated ACL reconstruction, the elimination of lateral thrust and preservation of articular cartilage of the medial compartment are of paramount importance to the future of these knees.
\end{abstract}

Keywords: Cruciate Ligament; Reconstruction; High Tibial Osteotomy; Varus Knees

Abbreviations: ACL: Anterior Cruciate Ligament; IKDC: International knee Documentation Committee Score; HTO: High Tibial Osteotomy; CT: Computed Tomography; WB: Touch Weight Bearing; ROM: Range of Motion

\section{Introduction}

Chronic ACL deficiency is considered an important factor in the development of degenerative changes and possible osteoarthritis of the knee joint [1]. This may be related to the alteration of knee kinematic function, repeated giving way or trauma to the articular cartilage in addition to the increased incidence of meniscus injuries. Therefore, early reconstruction of the ACL was suggested to avoid the development of other intra-articular pathologies [2,3]. To avoid deterioration of knee function, it is important to address other pathologies in the knee joint while performing ACL reconstruction such as meniscus repair or attention to chondral lesions. It may also be essential to correct deformities around the knee joint that may increase the load on one of the knee compartments or stretch the ACL graft. To stretch the line of thought, it is not uncommon for patients with varus knees and early degeneration of the articular cartilage to develop acute ACL injury with subsequent knee instability and aggravation of medial compartment symptoms of arthritis. The resulting argument here would be: should the ACL be reconstructed in these patients in isolation or as a combined procedure to simultaneously correct the pre-existing varus deformity? In this article, combined ACL reconstruction and HTO were performed for patients with deficient ACL and symptomatic varus deformities that were associated with medial compartment early degenerative changes and/or lateral thrust.

\section{Material and Methods}

Between May 2018 and April 2019, short term pilot study including 10 patients with ACL injuries and varus knees were treated. All cases were males; the mean age was 25 years (range, 20-40 years). The institutional review board approved the study before commencement and written consent was obtained from all patients. Regarding the inclusion criteria, all patients had symptomatic ACL deficiency with repeated attacks of giving way. The clinical examination of these patients revealed positive Lichtman and pivot shift tests. All patients had symptomatic varus knee alignment with medial joint line pain that was aggravated by long standing or walking for more than 15 minutes. The varus deformity in these patients varied from primary varus with no lateral joint laxity to double or triple varus according to Noyes' classification [4]. There was a dynamic increase of the deformity during the stance phase of gait and decrease of the deformity during the swing phase of gait. This dynamic frontal plane instability is known as varus thrust [5]. This was present in nine cases. 
All patients reported aggravation of their medial joint line pain following the knee trauma that resulted in ACL deficiency. The presence of widespread arthritic changes that involved different knee compartments with elimination of the medial joint space was considered an exclusion criterion. However, early degenerative changes with narrowing of the medial joint space on a standing X-ray were not considered exclusion criteria. Patients older than 40 years or those with tri-compartmental knee osteoarthritis, as well as patients with any degree of fixed flexion deformity were excluded. Similarly, patients with asymptomatic minor (5 degrees or less) of primary varus knee malalignment were not considered for the combined procedure. Prospective clinical and radiological data were recorded for all patients. Clinical evaluation assessed patients' symptoms, time since injury, changes of patients' symptoms over time, as well as clinical assessment for presence of lateral thrust, instability, points of tenderness, and the knee range of motion. The International Knee Documentation Committee score and patients' satisfaction were also recorded. Standing X-rays, magnetic resonance imaging, scans of the knees and CT scanogram were performed preoperatively. The degree of varus deformity (tibio-femoral angles), anatomical medial proximal tibial angles, arthritic changes within different compartments of the knee joint, the posterior tibial slope, and the angle of the opening wedge were measured from standing X-rays. X-rays were performed on the 2 nd postoperative day, then at 2 weeks, 6 weeks, 12 weeks, and 24 weeks postoperatively.

\section{Surgical Technique}

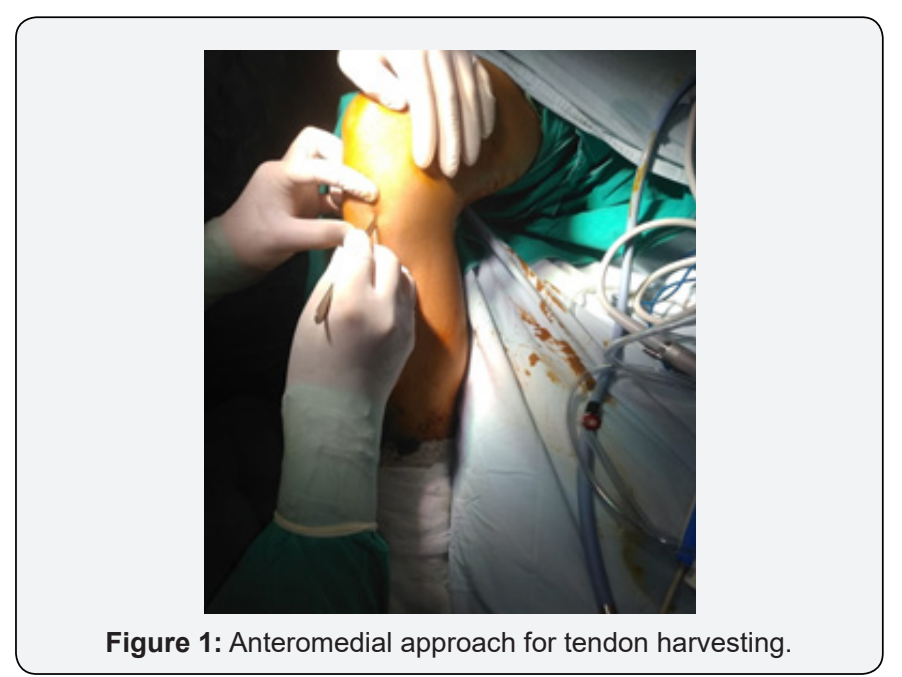

Operations were performed with patients placed in the supine position and tourniquet was applied to the upper thigh. Autogenous hamstring (gracilis and semitendinosus) tendons were harvested through anteromedial approach (Figure 1). Anatomical single bundle ACL reconstruction with preparation of the femoral tunnel through the anteromedial portal was the standard technique. The quadruple hamstring grafts were fixed on the femoral and acetabular sides using bioabsorbable interference screws (Absorbable interference screw, DePuy Mitek Inc, 325 Paramount Drive, Raynham, MA 02767, United States of America) that were of the same socket diameter on the femoral side and $1 \mathrm{~mm}$ larger than the tunnel diameter on the tibial side. Fixation of the graft on the femoral side was performed first, whereas fixation of the graft within the tibial tunnel was delayed until the HTO was completed to avoid any change of the graft tension following correction of the limb alignment. The tibial osteotomy was performed through the same approach under X-ray ( $\mathrm{C}$-arm) control. The medial surface of the proximal tibia was exposed with release of the medial collateral ligament. Subchondral k-wire was applied to protect tibial condyles during osteotomy. Two parallel K-wires were inserted along the plan of the osteotomy from distalemedial to proximalelateral direction (Figure 2). The osteotomy was performed just distal to the K-wires and started $1 \mathrm{~cm}$ distal to the cortical side of the ACL tibial tunnel at the metaphyseal diaphyseal junction (Figure 3). An oscillating saw was used to commence the osteotomy from medial to lateral direction while the patellar tendon was protected. The osteotomy was then completed by thin osteotomes through the medial and posterior cortices stopping short from the lateral cortex that was broken by valgus stress applied to the limb with a fulcrum at the level of the osteotomy. The mechanical axis of the lower limb was adjusted intra-operatively under X-ray control to ensure elimination of the varus deformity.

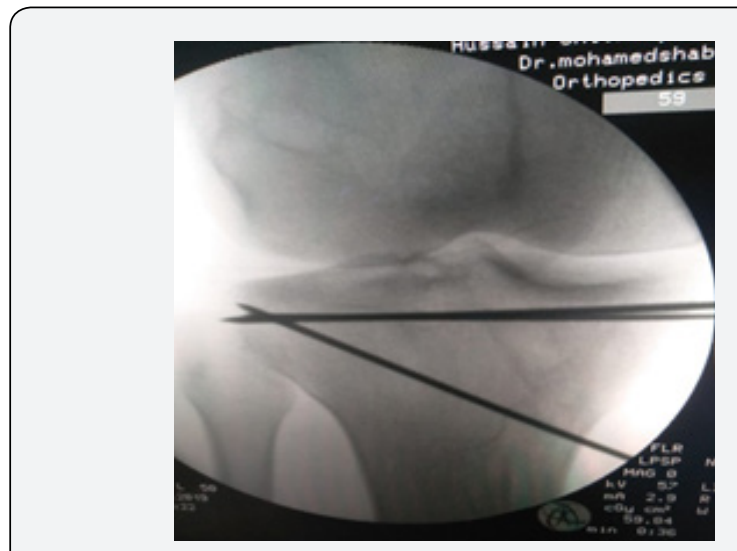

Figure 2: K-wires tip above the superior tibiofibular joint.

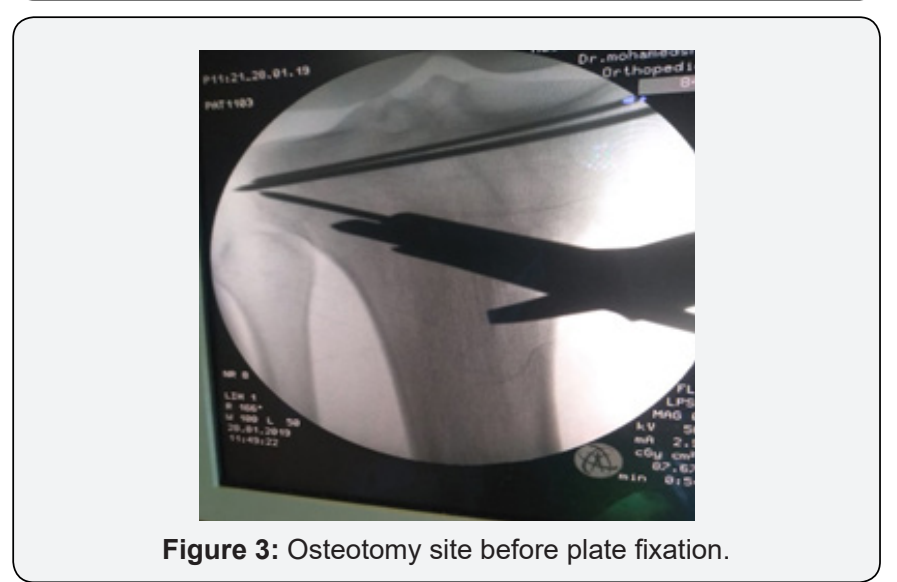


The osteotomy site was opened medially and maintained by laminar spreaders between the proximal and distal ends until fixation was completed by the plate and screws to the proximal and distal fragments (Figure 4). The posterior tibial slope was maintained to the preoperative values. Arthroscopic grading for the state of the articular cartilage over the medial compartment of the knee was recorded. Outer Bridge classification was used to give a grade for any degenerative changes in the articular cartilage. This is in addition to recording the integrity of the medial meniscus. Chondral lesions with loose cartilage flaps that may cause mechanical symptoms were removed. Similarly, complex meniscus tears were trimmed to a stable base. However, no specific cartilage or meniscal reconstructive procedures were performed. The postoperative rehabilitation protocol included application of an above knee splint for 2 weeks. This was followed by a hinged knee brace from the 3rd week with early quadriceps exercises and flexion to 30-degree flexion increased to 90 degree by the 4 th week. Then, ROM increased gradually to full ROM afterwards. Touch weight bearing (WB) started as early as when the patient was able to move out of bed. However, partial WB started at the 7th week and full WB was allowed from the 9 th week. A hinged knee brace was used for 12 weeks.

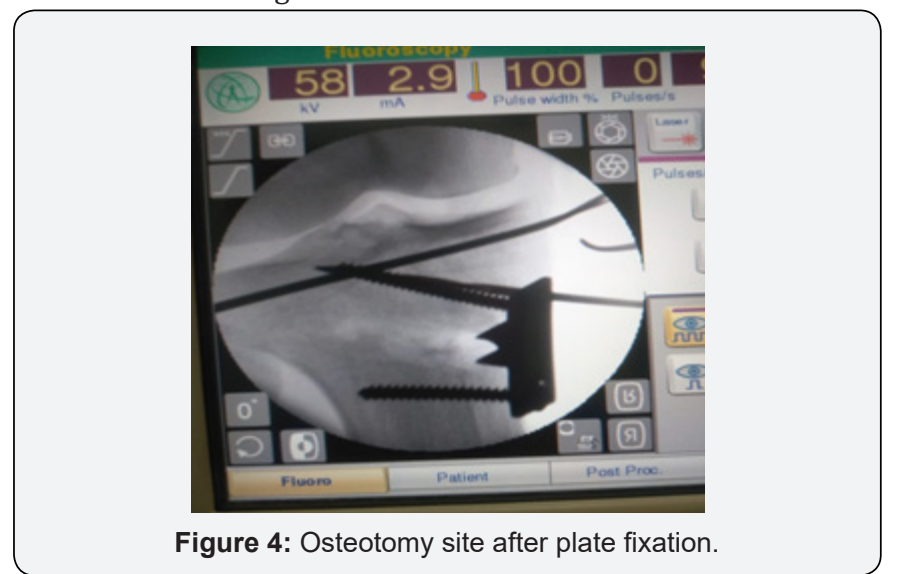

Statistical Analysis

Recorded data were analysed using the statistical package for social sciences, version 20.0 (SPSS Inc., Chicago, Illinois, USA). Quantitative data were expressed as mean \pm standard deviation (SD). Qualitative data were expressed as frequency and percentage.

\section{Results}

Ten patients with ACL deficiency and varus deformity of the knee had simultaneous anatomic single bundle ACL reconstruction and correction of their varus deformities. six patients were heavy manual workers, whereas the remaining four were regularly participating in leisure time sports activities. None of the patients was lost from the follow-up with an average follow-up period of 6 months (range, 3-12 months). The chief complaints of these patients preoperatively were a combination of knee instability and medial joint line pain. The average time from injury until surgical reconstruction was 9 months (range,
2-20 months). All patients reported worsening of their medial joint line pain after the knee trauma that caused the ACL injury and knee instability. Variable degrees of degeneration to the articular cartilage of the medial compartment of the knee were observed in this group of patients. five patients had grade 1 (softening of articular cartilage over the medial femoral condyle) whereas three others had grade 2 (fibrillation of the articular cartilage). Two patients had cartilage flaps $<10 \mathrm{~mm}$ that required debridement. Three patients required partial menisectomy with preservation of the meniscocapsular attachment. One patient had evidence of old healed meniscus root tear with minimal posterior displacement.

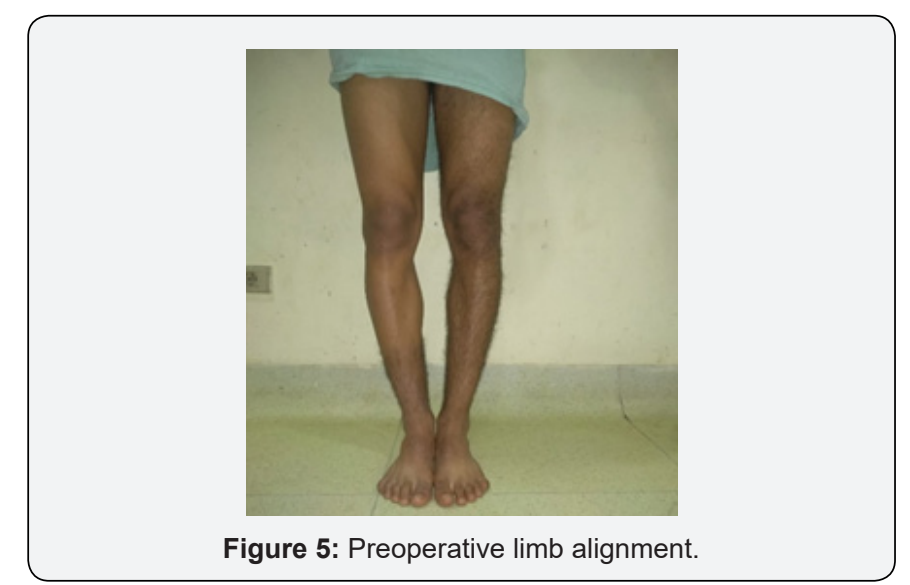

The average preoperative varus angle was 9 degrees (range, 5-12 degrees). All knees except one had full correction of their varus deformities to a neutral axis. The mean preoperative proximal medial tibial angle was 82 degree (range, 77-84 degree) whereas the mean postoperative angle was 89 degree (range, 87-92 degree). All knees had full correction of the varus deformity with no collapse at the osteotomy site (Figure 5). The average time for healing of the osteotomy site was 12 weeks (range 10-18 weeks). No healing problems were recorded in this study. All knees had positive Lichtman test (grades 2 to 3) and rotatory instability (positive pivot shift test grades 1 to 3 ) preoperatively. At the latest postoperative follow-up, all knees were stable with negative pivot shift test and anteroposterior laxity $<3 \mathrm{~mm}$. lateral thrust was present in nine knees. This lateral thrust has disappeared postoperatively. All patients had relief of medial knee joint pain. The mean IKDC score significantly improved from 37 to 78 postoperatively $(\mathrm{p}<0.05$ ). All patients returned to pre-injury level of activity, and all were fully satisfied with the procedure. The average tourniquet time for the combined procedure was 90 minutes. The operative time was almost equally split between the two procedures.

\section{Discussion}

ACL deficiency in the presence of varus alignment of the knee is not an uncommon finding. There is an ongoing debate on whether ACL reconstruction should be associated with correction of the varus deformity and if correction of the deformity is to be performed, should this be performed as sequential or a 
combined procedure? [6]. There is a direct relationship between varus malalignment and ACL tension 6 . In one cadaveric study, it was noted that when knees were loaded in increasing degrees of varus, tensile forces increased in the ACL, and when the ACL was resected, the same knees demonstrated increasing lateral opening with increased stresses [7]. Therefore, it may be better for the success of ACL reconstruction and knee overall function to simultaneously correct the varus deformity. In this series, the combined procedure of anatomic ACL reconstruction and open wedge HTO were performed on patients who had both instability and symptomatic varus deformity with a satisfactory outcome for both instability and medial joint line pain. The success achieved in this series would help in establishing some criteria for patient selection. It is therefore essential to place the emphasis on the presence of symptomatic varus along with the instability symptoms and signs. Hence, individuals with asymptomatic primary varus deformities without widening of the lateral joint space may not be ideal candidates of this combined procedure [810]. Previous reports suggested performing the combined ACL reconstruction along with correction of varus malalignment only in the presence of lateral thrust as it puts the reconstructed ACL under a high risk of failure [11]. It was also mentioned that varus malalignment in an ACL-deficient knee does not necessarily lead to a varus thrust, and therefore varus malalignment does not always require operative correction [8]. It could be argued that combining HTO with ACL reconstruction increases the surgical risk and may delay the rehabilitation process [9]. However, this was not observed in this series.

All patients went back to preoperative levels of activity with satisfactory pain relief and restored stability of the knee. These excellent results could be demonstrated by the absence of rotatory and anteroposterior instability as well as the significant improvement of the IKDC scores. Sagittal plane alignment of the knee should be considered when performing combined ACL reconstruction and valgus HTO. There is a positive correlation between exaggerated anterior tibial translation and increased posterior tibial slope [10]. For every 10 degrees increase in the posterior tibial slope, there is a corresponding $6 \mathrm{~mm}$ increase in the anterior tibial translation [11]. Dejour et al. [12] recommended reducing the tibial slope in ACL deficient knees if it exceeds 10 degrees. Similarly, Webb et al. [13] suggested that increased posterior tibial slope is associated with increased possibility for further ACL injury after ACL reconstruction.

The increased risk is more pronounced in those with a posterior tibial slope of 12 degrees. However, other authors deny the effect of changes of posterior tibial slope on ACL graft tension. On the contrary, in their cadaveric study Griffin et al. [14] concluded that increases in tibial slope do not affect anteriorposterior (A-P) translations or in situ forces in the cruciate ligaments. In this series, the postoperative posterior tibial slope angle was maintained in line with its preoperative values. Open wedge medial osteotomy was used in all patients in this series.
The open wedge technique avoids fibular osteotomy, dissection of the peroneal nerve, and detachment of tibialis anterior muscle [15]. This technique allowed restoration of the mechanical axis of the lower limb with only one incident of minimal collapse at the site of osteotomy. A simultaneous procedure is preferred over a staged procedure as it is a short-time procedure that does not increase the total surgical time by much, does not interfere with the tibial tunnel placement, and allows early recovery of the patients. The weakness of this study is related to the small sample size. However, the strict patients' selection criteria and close follow up of all patients provide a clear indication for the procedure and its expected outcome. In summary the combined procedure of ACL reconstruction and HTO offers a highly successful outcome in a special group of patients with symptomatic varus deformity and knee instability following ACL injury. The effect of this procedure on the development and progression of arthritic changes can only be observed through long-term evaluation.

\section{Conflict of Interest}

The author declared that no conflict of interest exists.

\section{References}

1. Wong JML, Khan T, Jayadev CS, Khan W, Johnstone D (2012) Anterior cruciate ligament rupture and osteoarthritis progression. Open Orthop J 6: 295-300.

2. Noyes FR, Basset RW, Grood ES, Butler DL (1980) Arthroscopy in acute traumatic hemoarthrosis of the knee. Incidence of anterior cruciate tears and other injuries. J Bone Joint Surg 62(5): 687-695.

3. Balkfors B (1982) The course of knee ligament injuries. Acta Orthop Scand 198: 1-99.

4. Noyes FR, Barber-Westin SD, Hewett TE (2000) High tibial osteotomy and ligament reconstruction for varus angulated anterior cruciate ligament-deficient knees. Am J Sports Med 28(3): 282-296.

5. Chang A, Hochberg M, Song J, Dunlop D, Chmiel JS, et al. (2010) Frequency of varus and valgus thrust and factors associated with thrust presence in persons with or at higher risk for knee osteoarthritis. Arthritis Rheum 62(5): 1403-1411.

6. Boss A, Stutz G, Oursin C, Gächter A. et al. (1995): Anterior cruciate ligament reconstruction combined with valgus tibial osteotomy. Knee Surg Sports Traumatol Arthrosc 3: 187-191.

7. Markolf KL, Burchfield DM, Shapiro MM, Shepard MF, Finerman GA, et al. (1995): Combined knee loading states that generate anterior cruciate ligament forces. J Orthop Res 13(6): 930-935.

8. Van de Pol GJ, Arnold MP, Verdonschot N, van Kampen A (2009): Varus alignment leads to increased forces in the anterior cruciate ligament. Am J Sports Med 37(3): 481-487.

9. Kim SJ, Moon HK, Chun YM, Chang WH, Kim SG, et al. (2011) Is correctional osteotomy crucial in primary varus knees undergoing anterior cruciate ligament reconstruction? Clin Orthop Relat Res 469(5): 14211426.

10. Jackson JP, Waugh W (1960) Tibial osteotomy for osteoarthritis of the knee. Proc R Soc Med 53(10): 888.

11. Dejour H, Walch G, Deschamps G, P Chambat (1987) Arthrose du genou sur laxite chronique anterieure. Rev Chir Orthop Reparatrice Appar Mot 73: 157-170. 
12. Dejour H, Neyret P, Boileau P, Donell ST (1994): Anterior cruciate reconstruction combined with valgus tibial osteotomy. Clin Orthop Relat Res 299: 220-228.

13. Webb JM, Salmon LJ, Leclerc E, Pinczewski LA, Roe JP (2013) Posterior tibial slope and further anterior cruciate ligament injuries in the anterior cruciate ligament-reconstructed patient. Am J Sports Med 41(12): 2800-2804.
14. Giffin JR, Vogrin TM, Zantop T, Woo SL, Harner CD (2004) Effects of increasing tibial slope on the biomechanics of the knee. Am J Sports Med 32(2): 376-382.

15. Luites JW, Brinkman JM, Wymenga AB, van Heerwaarden RJ et al. (2009) Fixation stability of opening versus closing wedge high tibial osteotomy: a randomized clinical trial using radiostereometry. J Bone Joint Surg Br 91(11): 1459-1465.

\section{Your next submission with Juniper Publishers will reach you the below assets}

- Quality Editorial service

- Swift Peer Review

- Reprints availability

- E-prints Service

- Manuscript Podcast for convenient understanding

- Global attainment for your research

- Manuscript accessibility in different formats ( Pdf, E-pub, Full Text, Audio)

- Unceasing customer service

Track the below URL for one-step submission https://juniperpublishers.com/online-submission.php 